\title{
More on the relation between division and multiplication in simple arithmetic: Evidence for mediation of division solutions via multiplication
}

\author{
JO-ANNE LEFEVRE and JASON MORRIS \\ Carleton University, Ottawa, Ontario, Canada
}

\begin{abstract}
Adults $(N=32)$ solved simple multiplication (e.g., $8 \times 7)$ and corresponding division problems (e.g., $56 / 8$ ). Self-reports of solution processes were given by half of the participants. Latency patterns and error rates were closely related across operations and were similar in self-report and no-report conditions. Solution of division problems, however, facilitated solution of multiplication problems more than the reverse. On large division problems, participants reported that they "recast" problems as multiplication (e.g., $56 / 8$ as $8 \times ?=56$ ). These results support the hypothesis that multiplication and division are stored in separate mental representations but that solution of difficult division problems sometimes involves access to multiplication.
\end{abstract}

Research on simple arithmetic problems provides important information about the mental representation and processing of basic numerical knowledge (reviewed by Ashcraft, 1995; see also LeFevre, Bisanz, et al., 1996; McCloskey \& Macaruso, 1994). Although a considerable amount of research has accumulated on the solution of addition and multiplication problems, subtraction and division have received little attention (cf. Campbell, 1997; Geary, Frensch, \& Wiley, 1993; Rickard \& Bourne, 1996; Rickard, Healy, \& Bourne, 1994). In the present study, we examined the relation between performance of simple multiplication (e.g., $6 \times 9=$ ?) and simple division (e.g., $54 / 6=$ ?) problems: Comparisons across operations are useful for testing the generality of theoretical accounts of arithmetic solutions (e.g., Campbell, 1995; Geary, Widaman, \& Little, 1986; Rickard et al., 1994).

Following earlier work by Rickard and Bourne (1996; Rickard et al., 1994), Campbell (1997) outlined two hypotheses about the representation of multiplication and division knowledge. First, according to the common representation hypothesis, a single network exists that is accessed differentially according to the specified operation. Second, according to the independent representation hypothesis, two separate mental networks exist, one for each

This research was funded by the Natural Sciences and Engineering Research Council of Canada. Portions of the results were presented at the annual meeting of the Canadian Society for Brain, Behaviour, and Cognitive Science in Montreal, August 1996. The authors thank Karen Daley and Chris Herdman for their helpful comments on a previous version of this article. The data for latencies, percentage of errors, and percentage use of solution procedures on each problem are available on the Web at http://www.carleton.ca/ jlefevre/papers/l\&m 99 app.htm or from the first author. Correspondence should be addressed to J.-A. LeFevre, Department of Psychology, Carleton University, Ottawa, ON, Canada K1S 5B6 (e-mail: jo-anne_lefevre@carleton.ca). operation. The goals of the present research were to compare performance across multiplication and division in order to explore whether multiplication and division are contained in independent mental representations or in a common representation and to examine the solution processes used by individuals solving division problems.

Campbell (1997) found support for the common representation hypothesis in that patterns of latencies and errors on multiplication and division problems were very similar. The operations showed similar effects of problem size (i.e., latencies and errors increased with the size of the operands), similar advantages for ties (e.g., $3 \times 3 ; 64 \div 8$ ) over nonties (e.g., $3 \times 4 ; 63 \div 9$ ), and similar advantages for problems with operands of 5 (e.g., $5 \times 6 ; 40 \div 8$ ) relative to problems that do not have operands of 5 (e.g., $4 \times$ $8 ; 32 \div 4$ ). Effects of problem size, ties, and 5-operand problems are well established for multiplication (e.g., Campbell \& Graham, 1985; LeFevre, Bisanz, et al., 1996; Miller, Perlmutter, \& Keating, 1984) but had not been previously reported for division. Furthermore, the effects of problem size, tie, and 5-operand status on latencies or percent errors were comparable for multiplication and division in that they were all statistically significant (see Table 2 of Campbell, 1997). Finally, the correlations between latencies and errors on each problem across operations were high [e.g., $r(34)=.82$ between latencies on division and multiplication problems]. These findings are necessary but not sufficient evidence that a common representation was used to solve division and multiplication problems.

Campbell (1997) also found some evidence that multiplication and division involved independent representations. Campbell (1997) examined effects of error priming across operations. Error priming refers to the finding that errors to multiplication problems in a speeded produc- 
tion task (e.g., $4 \times 6=32$ ) are often the correct answers to problems that have recently been presented (i.e., within the last 10 trials; Arbuthnott \& Campbell, 1996; Campbell, 1991; Campbell \& Clark, 1989; Campbell \& Tarling, 1996). ${ }^{1}$ Campbell (1997) found that recently solved multiplication problems produced error priming for multiplication and recently solved division problems produced error priming for division. Consistent with the independent representation hypothesis, recently solved multiplication problems did not produce error priming for division, in accord with the view that they were solved via different cognitive processes. Contrary to the independent representation hypothesis, however, earlier division problems produced error priming on multiplication problems, indicating that solving a division problem involved some sort of access to multiplication information. Symmetrical error priming across operations would be strong evidence in favor of a common representation, but the asymmetrical priming that was found could be explained more readily under the assumption of independent representations because error priming is extremely specific. For example, a problem in verification format (e.g., $6 \times 5=30$ ) does not produce much error priming for production (e.g., $6 \times 7=$ ?), and vice versa (Campbell \& Tarling, 1996).

To account for the obtained patterns of error priming, Campbell (1997) suggested that participants may have checked division answers after solution by accessing the associated multiplication fact. Thus, solution of $54 \div 6=$ 9 may have been verified by accessing $6 \times 9=54$. The assumption that checking occurred only for division is necessary to account for the asymmetrical nature of error priming. On this view, Campbell (1997) concluded that there was some evidence in support of the independent representation hypothesis, although he also suggested that multiplication knowledge may somehow mediate solution of division problems via a common representation.

Rickard and Bourne (1996) and Rickard et al. (1994) also reported evidence in support of an independent representation model. They found that extended practice of specific multiplication and division facts did not produce transfer to related facts across operations. For example, practice of $6 \times 8$ did not facilitate latencies to $48 \div 6$, or vice versa. Furthermore, practice of one form of division problem (e.g., $48 \div 6$ ) did not transfer to the related form (e.g., $48 \div 8$ ). To account for these results, Rickard et al. (1994) proposed an identical elements model of arithmetic solutions such that items that share both operands and the operation sign (e.g., $6 \times 8$ and $8 \times 6$ ) are processed by the same representation, whereas items that do not share all three elements (e.g., $48 \div 6$ and $48 \div 8$ ) are processed via different representations. Hence, according to the identical elements model, $8 \times 6$ and $48 \div 6$ would be processed in independent mental structures.

In the present research, we hypothesized that division and multiplication facts are stored in independent representations but that solution of division problems often involves access to multiplication knowledge. Campbell's
(1997) suggestion that multiplication may be used to check division answers after retrieval has occurred was an attempt to reconcile the evidence for the common representation hypothesis with that for the independent representation hypothesis. Campbell (1997) does not offer an explanation, however, of why a multiplication check is used or why it might occur after a solution was produced. Alternatively, we propose that access to multiplication knowledge may occur as part of the solution of division problems. For example, an individual presented with a problem such as $56 \div 8$ may solve the problem by activating the corresponding multiplication problem (e.g., $8 \times$ $?=56$ ). The use of such a procedure could account for some aspects of Campbell's (1997) data, including the latency and error advantage of multiplication over division. To account for the asymmetrical priming, we propose that activation of multiplication during division problems involves direct access to the multiplication representation and thus facilitates later multiplication solutions. In contrast, although activation of multiplication problems may facilitate division to some extent, it does not produce direct activation of the relevant division representation, and, thus, only a small amount of priming would be expected when multiplication problems precede division.

Recent research has shown that arithmetic solutions that refer to another operation are commonly reported on addition, multiplication, and subtraction problems (Geary, 1996; Geary et al., 1993; Geary \& Wiley, 1991; LeFevre, Bisanz, et al., 1996; LeFevre, Sadesky, \& Bisanz, 1996). Such multiple-step procedures are most common on the more difficult problems (i.e., those with larger operands). On smaller and easier problems, direct retrieval from memory is the more typical solution approach. For example, on approximately $30 \%$ of trials, adults reported solutions such as $8 \times 8+8$ for the problem $8 \times 9$ (LeFevre, Bisanz, et al., 1996). Hence, we suggest that an independent representation may be accessed directly to solve easy division problems (e.g., $6 \div 3$ or $12 \div 2$ ) but that solution of larger, more difficult problems (e.g., $56 \div 8$ ) may often involve interrogation of multiplication knowledge.

To examine the hypothesis that solution of division problems sometimes involved multiplication, half of the participants in the present experiment provided selfreports of their solution processes on each problem immediately after reporting the answer (Geary, 1996; Geary et al., 1993; Geary \& Wiley, 1991; LeFevre, Bisanz, et al., 1996; LeFevre, Sadesky, \& Bisanz, 1996). To address the possibility that the requirement to produce self-reports may influence patterns of performance, the other participants did not give self-reports. Performance in the two conditions was compared in order to examine whether selfreports influenced patterns of latencies or errors.

To examine the hypothesis that multiplication and division are stored in independent representations, we blocked problems by operation and manipulated presentation order. Thus, half of the participants solved multiplication problems first and division problems second, and the other half did the reverse. We hypothesized that 
individuals who solved division before multiplication should show greater benefits on the second block of trials than those who solved multiplication before division. Such asymmetry of transfer would support the hypothesis that multiplication and division involve separate representations but that solution of division problems sometimes involve direct access to the multiplication representation.

We also hypothesized that access to multiplication knowledge on division trials would occur mainly on the largest, most difficult division problems because the division representation is most likely to be incomplete for those problems. Hence, we predicted that transfer from division to multiplication would be greater on large operand problems (e.g., 72/9) than on small operand problems (e.g., 12/4). Accordingly, we also expected that participants would be more likely to report accessing multiplication knowledge on division trials for larger problems than on division trials for smaller problems.

\section{METHOD}

\section{Participants}

Thirty-three undergraduate psychology students ( 17 males and 16 females) participated in partial fulfillment of a course requirement. One participant had extremely slow latencies (averaging greater than $2 \mathrm{sec}$ ), and, thus, his data were not analyzed.

\section{Materials}

The participants solved the 81 multiplication problems as defined by all possible combinations of integers from 1 to 9 (i.e., $1 \times$ 1 to $9 \times 9$ ) and the 81 complementary division problems (i.e., $1 / 1$ to $81 / 9$ ). Each operation (i.e., multiplication and division) was presented as a block of trials. Complementary pairs of problems (e.g. $8 \times 6$ and $6 \times 8$ or $56 / 8$ and 56/7) were presented in different halves of each block. Ten practice trials were selected for each operation such that each of the nine operands appeared at least once. These problems were then placed near the end of the experimental trials in order to minimize specific practice effects.

The participants also completed an assessment of calculation fluency: the subtraction-multiplication and addition subtests of the French Kit (French, Ekstrom, \& Price, 1963). The subtractionmultiplication subtest involves rows of two-digit subtraction problems alternating with multidigit multiplication problems. The addition subtest involves three-term addition problems with multidigit addends. Each subtest consists of 2 pages. The participants also completed a questionnaire on the strategies they used when solving addition, subtraction, and multiplication problems, and their experiences with mathematics. ${ }^{2}$

\section{Apparatus \\ The simple multiplication and division problems were presented on a Samsung model SM 125FA7 monitor connected to an IBM- type 80286 computer. Responses were made through a Sharpe LM6 boom microphone, which was interfaced with a Soundblaster $\mathrm{I} / \mathrm{O}$ board. Latencies were recorded with a Digitek I/O board and were accurate to the nearest millisecond. \\ Procedure \\ Presentation order of operations was counterbalanced across sex and report condition (self-report vs. no-report). Before starting the computer trials, the participants were instructed to respond to each problem as quickly as possible, by stressing that "everyone makes}

mistakes" and that it is "important that you go quickly." The participants in the self-report condition were also told that, after they had answered each problem, they were to explain to the researcher how they got their answer. It was suggested that, on some trials, they may have "just remembered" the answer or "just known" it, and, on other trials, they may have "changed or simplified the problem" in order to answer it. They were asked to try to describe to the researcher everything they did to get their answer.

On each trial, an asterisk (*) was presented for $1 \mathrm{sec}$ in the center of the screen. The asterisk then flashed off and on twice over a 1 -sec interval. On what would have been the third appearance of the asterisk, the problem was presented, with the operation sign ( $\times$ or $/)$ at the location of the asterisk. This presentation was identical to that described by Campbell $(1994,1995)$. The problem remained on the screen until the participant gave a verbal response or until $5 \mathrm{sec}$ had elapsed. The experimenter recorded the response, and it was scored by the computer. In the no-report condition, the next trial was initiated $500 \mathrm{msec}$ after the experimenter recorded the participant's answer.

In the self-report condition, the prompt "How did you solve the problem?" appeared on the screen. After listening to the participant's description, the experimenter indicated whether the participant reported using retrieval or some other procedure. Retrieval was recorded for responses such as "just knew it," "memory," "I don't know," or "guessed." The next trial was initiated $500 \mathrm{msec}$ after the experimenter entered the participant's solution. Procedures were subsequently classified by an independent coder for each operation based on the information recorded by the experimenter.

Before completing the experimental trials, the participants solved 10 practice trials with feedback about accuracy. After the practice trials, the speed instructions were reemphasized, and then the experimental trials were presented. No feedback about accuracy was given on experimental trials. In each block, the message "Rest now. You are halfway through." appeared after $\mathbf{4 0}$ trials. After completing both division and multiplication problems, the participants filled out the questionnaire. Finally, they completed the French Kit tasks (approximately $10 \mathrm{~min}$ ). Time to complete the whole experiment ranged from 50 to $70 \mathrm{~min}$.

\section{RESULTS}

Unless otherwise indicated, an alpha level of .05 was used in all analyses. For multiplication problems (e.g., $3 \times$ $4=12$ ), the first two numbers are referred to as the operands, and the answer is referred to as the product. For division problems (e.g., $56 / 8=7$ ), the first number is referred to as the dividend, the second as the divisor, and the third as the quotient. In the present paper, when direct comparisons were made between multiplication and division, the divisor and the quotient were referred to as the operands.

\section{Calculation Fluency}

To ensure that overall levels of calculation skill were similar across report groups, total scores on the French Kit fluency test were compared. Fluency scores for participants who gave self-reports $(M=92, S D=22)$ were not significantly different from those who did not report their solutions $(M=90, S D=20)$. Given these data, we would not expect the self-report and no-report groups to differ in overall latencies or percentage of errors on the experimental trials. 


\section{Analyses of Latencies and Percent Errors}

Of the total 5,184 trials, $4.2 \%(n=218)$ were invalid because of extraneous vocalizations or equipment failures. These trials were omitted from the analyses.

Participant-based analyses. Latencies on problems with operands of 1 were analyzed separately from the remaining problems so that analyses of the other problems could be compared directly with those reported by Campbell (1997). Errors on problems with operands of 1 were not analyzed because only 8 errors were made (on the 1,030 valid trials). Median latencies on problems with operands of 1 were analyzed in a 2 (operation: multiplication, division) $\times 2$ (condition: self-report, no-report) $\times$ 2 (order: multiplication-division, division-multiplication) analysis of variance (ANOVA), with repeated measures on the first factor. The participants solved multiplication problems faster than division problems (699 vs. $854 \mathrm{msec})$ $\left[F(1,28)=42.77, M S_{\mathrm{e}}=9,027\right]$. No other main effects or interactions approached significance $(p s>.30)$. Thus, latencies on problems with operands of 1 were fast and accurate, and the requirement to produce self-reports did not appear to influence performance.

For problems with operands of 2-9, problem size was indexed by operand family (Campbell, 1994). Median latencies and percent errors were calculated across the maximum of 15 problems in each operand family; hence, each individual latency contributed to two different operand families. Median correct latencies and percent errors were analyzed in 2 (order: multiplication-division, divisionmultiplication) $\times 2$ (report condition: self-report, noreport) $\times 2$ (operation: multiplication, division) $\times 8$ (operand family) ANOVAs, with repeated measures on the last two factors.

Multiplication problems were solved more quickly than division problems $(1,006$ vs. $1,117 \mathrm{msec})[F(1,28)=$ $\left.16.62, M S_{\mathrm{e}}=94,653\right]$ and with fewer errors $(7.4 \%$ vs. $11.0 \%)\left[F(1,28)=4.88, M S_{\mathrm{e}}=181\right]$, replicating the pattern across operations found by Campbell (1997). As expected, there was a substantial effect of problem size in that latencies and errors generally increased with operand family $\left[F \mathrm{~s}(7,196)=35.47\right.$ and $14.16, M S_{\mathrm{e}} \mathrm{s}=26,912$ and 78 , respectively]. Nonlinearities in the pattern of latencies by operand family were also evident. As has been reported for multiplication, problems with operands of 5 showed the typical latency and error advantages relative to other problems of a similar magnitude (Campbell, 1994, 1995; Campbell \& Graham, 1985; LeFevre, Bisanz, et al., 1996). Other deviations from a strictly linear increase with operand family were observed. According to the $95 \%$ confidence interval for the family effect $(41 \mathrm{msec})$, problems with operands of $2(879 \mathrm{msec})$ were faster than all others. Problems with operands of $3(954 \mathrm{msec})$ and $5(986 \mathrm{msec})$ did not differ but were faster than problems with operands of 4 $(1,047 \mathrm{msec})$. Problems with operands of $6(1,098 \mathrm{msec})$ or $7(1,084 \mathrm{msec})$ were not different but were slower than problems with operands of 4 . Problems with operands of $8(1,225 \mathrm{msec})$ or $9(1,220 \mathrm{msec})$ were slowest but not different from each other. Campbell (1997) found similar advantages for 5-operand problems and similar nonlinearities across operand families (see his Figures 1 and 2, p. 1145).

We hypothesized that solving division problems often involves accessing multiplication knowledge, whereas solving multiplication problems does not involve direct access to division representations. Consistent with this hypothesis, operation and presentation order interacted $\left[F(1,28)=4.93, M S_{\mathrm{e}}=94,653\right]$. The multiplication latencies of participants who solved multiplication problems after division were $83 \mathrm{msec}$ faster than those of participants who solved multiplication first ( $965 \mathrm{vs.} 1,048 \mathrm{msec}$ ). In contrast, the latencies of participants who solved division problems after multiplication were only $37 \mathrm{msec}$ faster than those of the participants who solved division first $(1,099$ vs. $1,136 \mathrm{msec})$. Thus, solving a block of division problems facilitated subsequent multiplication performance more than solving multiplication problems facilitated division.

The three-way interaction of operand family, operation, and order for latencies is shown in Figure $1[F(7,196)=$ $\left.2.43, M S_{\mathrm{e}}=13,590\right]$. The pattern of results for this interaction provides further support for the view that solving division problems had a substantial priming effect on multiplication. Consider the left panel of Figure 1, which shows the latencies for participants who solved multiplication problems first and division problems second. Both operations showed similar increases with operand family (i.e., problem size), and both showed the characteristic advantage for 5-operand problems. According to the $95 \%$ confidence interval $(58 \mathrm{msec})$, multiplication was faster than division for the 2-, 3-, 4-, and 8-operand families. On the 5-, 6-, 7-, and 9-operand families, however, the differences between operations were not significant. Thus, the advantage of multiplication over division was not constant across problem size when multiplication was presented first.

The right panel of Figure 1 shows the latencies for participants who solved division problems first and multiplication problems second. Multiplication was significantly faster than division for all operand families, suggesting that priming of multiplication facts occurred when division problems were solved first. Furthermore, the problem size effect in multiplication was substantially attenuated when multiplication was solved second (right panel). Although multiplication performance was similar on 2-, 3 -, and 5-operand problems in both presentation orders, multiplication latencies of participants who solved multiplication after division (right panel) were significantly faster for 4-, 6-, 7-, 8-, and 9-operand problems than those of participants who solved multiplication before division (left panel). Prior exposure to division problems appeared to facilitate the most difficult multiplication problems. In contrast, latencies on division problems were similar in both presentation orders. In summary, the patterns of performance across presentation orders and 

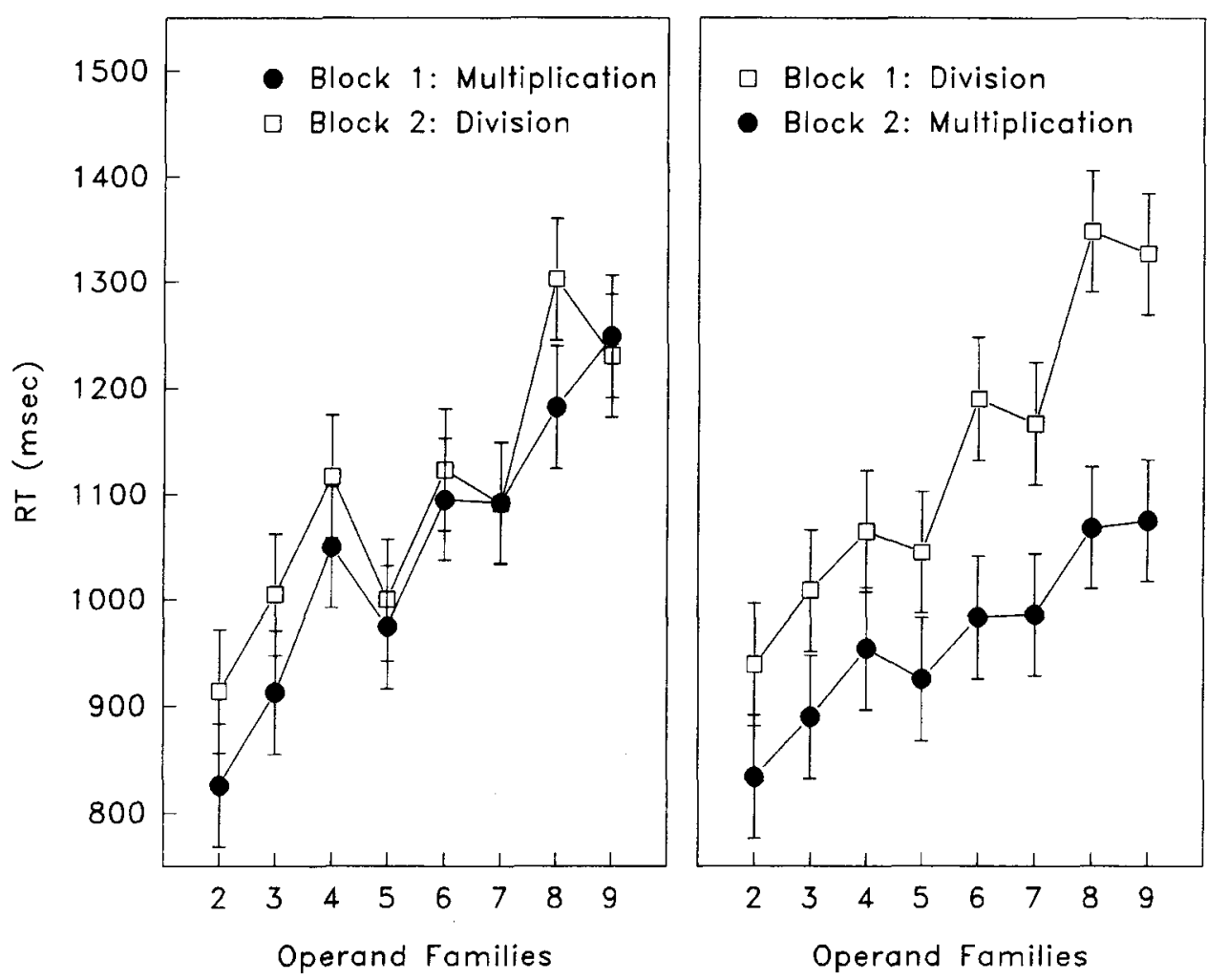

Figure 1. Mean median latencies (in milliseconds) by operand families for multiplication and division problems (with $95 \%$ confidence intervals). Data in the left panel are from those participants who solved multiplication first and division second. Data in the right panel are from those participants who solved division first and multiplication second.

operand families support the hypothesis that solution of division problems often involves access to associated multiplication knowledge.

Self-reports did not influence latencies, in that there were no significant effects of report condition. Thus, the present findings suggest that self-reports of solutions do not alter either overall performance or patterns of latencies for multiplication or division problems. For percent errors, however, there was a trend for no-report participants to make more errors than those who gave self-reports $(11.0 \%$ vs. $6.5 \%)\left[F(1,28)=3.86, M S_{\mathrm{e}}=668, p=.059\right]$. This effect is qualified by the three-way interaction of report condition, family, and operation $[F(7,196)=2.08$, $\left.M S_{\mathrm{e}}=46\right]$. Tests of simple effects indicated that there were no significant differences between self-report and no-report conditions for any problem family for either multiplication or division, suggesting that there was considerable variability across individuals. ${ }^{3}$

In summary, the participant-based analysis indicated that division problems were solved more slowly than multiplication problems. Solving a block of division problems, however, facilitated subsequent multiplication performance more than the reverse order of operations, in support of the hypothesis that solution of division problems frequently involves access to multiplication. As predicted, this priming effect was greater on the larger, more difficult multiplication problems (see Figure 1), suggesting that access to multiplication knowledge is most likely to occur on more difficult division problems.

Problem-based analyses. In previous research, regression analyses of item latencies collapsing across participants have been used to examine problem size effects (e.g., Campbell, 1995, 1997; LeFevre, Bisanz, et al., 1996; Miller et al., 1984). Therefore, to allow for direct comparisons across studies, median latencies and percent errors were calculated for each problem. Correlations among these dependent measures and each of the standard structural variables are shown in Table 1 (i.e., product or dividend, maximum operand, tie, and 5-operand status). The patterns of correlations among the dependent measures were generally similar to those obtained by Campbell (1997). Latencies and percent errors on division and multiplication were highly intercorrelated, and performance across operations was also very similar. Correlations between structural variables and latencies or percent errors were consistent with those obtained by Campbell (1997). The only obvious discrepancy involved the correlations between 5-operand status and latencies, which were lower in the present data than in Campbell (1997). ${ }^{4}$

Table 2 shows multiple regressions of latencies and percent errors for each operation on the three structural pre- 
Table 1

Problem-Based Correlations Among Latencies (Median RT in Milliseconds), Percent Error (\%Err), and Structural Variables for Multiplication and Division

\begin{tabular}{|c|c|c|c|c|c|c|c|}
\hline & \multicolumn{3}{|c|}{ Dependent Measures } & \multicolumn{4}{|c|}{ Structural Variables } \\
\hline & $\mathrm{RT}_{\text {div }}$ & $\%$ Err $_{\text {mult }}$ & $\% \mathrm{Err}_{\mathrm{div}}$ & Max & Product & 5-operand & Tie \\
\hline $\begin{array}{l}\mathrm{RT}_{\text {mult }} \\
\mathrm{RT}_{\text {div }} \\
\% \mathrm{Err}_{\text {mult }} \\
\% \text { Err }_{\text {div }}\end{array}$ & .87 & $\begin{array}{l}.72 \\
.69\end{array}$ & $\begin{array}{l}.69 \\
.69 \\
.57\end{array}$ & $\begin{array}{l}.61 \\
.66 \\
.48 \\
.54 \\
\end{array}$ & $\begin{array}{l}.64 \\
.65 \\
.53 \\
.51 \\
\end{array}$ & $\begin{array}{l}.14 \\
.20 \\
.32 \\
.27 \\
\end{array}$ & $\begin{array}{l}.18 \\
.21 \\
.11 \\
.20\end{array}$ \\
\hline
\end{tabular}

Note- Correlations between dependent measures and structural variables were calculated on all 64 problems. Correlations among betweenoperation variables (in italies) were calculated on the 36 items remaining after collapsing across both orders of operands for multiplication (e.g., $5 \times 9$ and $9 \times 5$ ) and divisors and quotients for division (e.g., $45 / 9$ and $45 / 5$ ). Structural variables are maximum operand (Max), product (dividend for division), 5-operand status ( -.5 for 5-operand problems, .5 for other problems), and tie status ( -.5 for tie problems, and .5 for nonties). Correlations greater than .25 are significant at $p<.05$, two-tailed.

dictors simultaneously. Multiple regressions are useful because the unique contribution of each predictor can be evaluated. In the present data, the large effect of problem size may overshadow the contribution of other variables. Consistent with this view, although the simple correlations for 5-operand and tie status were not significant for latencies, the 5-operand and tie advantages were significant in three of four cases in the multiple regressions (see Table 2). ${ }^{5}$ In general, the results of the multiple regression analyses were very similar for multiplication and division, in terms of both the size of the coefficients and the percentage of variance accounted for by the set of predictors. As concluded by Campbell (1997), problembased analyses suggest a close correspondence between the solution of multiplication and division problems.

In general, the coefficients shown in Table 2 are also similar to those reported for the simple regressions by Campbell (1997), with the exception that the coefficients for product (dividend) in latencies are larger in the present research (see also LeFevre, Bisanz, et al., 1996). The problem size effect may be smaller in the data presented by Campbell because he trimmed the slowest latencies from the data (treating latencies greater than $3 S D$ from the grand mean for each problem as outliers). If these outliers occurred mainly on the larger problems, this trimming procedure would presumably affect the mean latencies for larger problems more than those for smaller problems. To address this possibility, we analyzed division latencies reported across problems by Campbell (1997, his Appendix B) with ours for division problems in a 2 (experiment: Campbell, present) $\times 2$ (problem size: small, large) ANOVA, with repeated measures on the first factor. (Small problems had dividends less than 26 , and large problems had dividends greater than 26.) In this analysis, there were significant effects of experiment $\left[F(1,62)=19.70, M S_{\mathrm{e}}=\right.$ $5,282]$, problem size $\left[F(1,62)=35.8, M S_{\mathrm{e}}=37,411\right]$, and the interaction $\left[F(1,62)=29.04, M S_{\mathrm{e}}=5,282\right]$. On small problems, latencies were similar in the present experiment to those reported by Campbell ( 970 vs. $983 \mathrm{msec}$ ). On large problems, however, latencies in the present experiment were slower than those reported by Campbell $(1,244$ vs. $1,118 \mathrm{msec}$ ). Thus, the differences across experiments appear on larger, rather than on smaller, problems and do not reflect generally slower latencies in the present experiment relative to Campbell (1997).

In summary, multiplication and division performance across problems was highly correlated, and latencies for both operations showed very similar relations to structural variables, such as problem size and 5-operand status. Thus, with problem size operationalized as a linear variable, performance on multiplication and division problems appears to be very similar. As noted by Campbell (1997), these results are necessary but not definitive evidence for the common representation hypothesis.

\section{Self-Reports of Procedure Use}

One goal of obtaining self-reports of solution procedures was to examine the hypothesis that solution of division problems often involved access to multiplication. We considered two other issues with respect to selfreported solutions of multiplication and division problems. First, were patterns of self-reports for multiplication similar to those found by LeFevre, Bisanz, et al. (1996)? Second, did self-reports for division follow a similar pattern as has been observed in other operations-namely, more frequent use of procedures other than direct retrieval on more difficult problems?

Multiplication. For multiplication, nonretrieval responses could be categorized in a similar way to that used by LeFevre, Bisanz, et al. (1996), including the categories (1) repeated addition (e.g., $2 \times 3=3+3$ ), (2) series (e.g., $3 \times 5=5,10,15),(3)$ derived facts (e.g., $6 \times 9=6 \times 10$ -9 ), and (4) other (e.g., nines trick, rhyming as in $6 \times$ $8=48$ ). However, an additional category was necessary. Some participants reported visualizing the multiplication table in order to access the answer. In LeFevre, Bisanz, et al. (1996), visualization was reported very infrequently and thus was included in the "other" category. In the present research, however, 7 of the 16 participants in the selfreport condition mentioned visualization of the table of multiplication facts, and 4 reported using it frequently (on $86 \%, 50 \%, 42 \%$, and $39 \%$ of valid trials).

Table 2

Unstandardized Coefficients for Multiple Regressions of Median Latencies (in Milliseconds) and Percent Errors on Product (Dividend for Division), 5-Operand, and Tie

\begin{tabular}{lccccc}
\hline \multirow{2}{*}{$\begin{array}{l}\text { Dependent } \\
\text { Measure }\end{array}$} & & \multicolumn{4}{c}{ Predictor Variables } \\
\cline { 2 - 6 } & Intercept & Product & 5 -operand & Tie & $R^{2}$ \\
\hline $\mathrm{RT}_{\text {mult }}$ & $663.1^{* *}$ & $8.0^{* *}$ & 62.3 & $174.8^{* *}$ & $.49^{* *}$ \\
$\mathrm{RT}_{\text {div }}$ & $787.6^{* *}$ & $7.5^{* *}$ & $87.4^{*}$ & $188.9^{* *}$ & $.53^{* *}$ \\
$\%_{\text {Err }}$ & -3.6 & $0.24^{* *}$ & $6.1^{* *}$ & $5.2^{\mathrm{a}}$ & $.40^{* *}$ \\
\%Err $_{\text {div }}$ & -2.3 & $0.25^{* *}$ & $5.5^{*}$ & $7.8^{* *}$ & $.39^{* *}$ \\
\hline
\end{tabular}

Note -5 -operand problems were coded as -.5 and other problems as .5 . Tie problems were coded as -.5 and nonties as .5 . ${ }^{\mathrm{a}} \mathrm{a}=.055$. " $p<.05 . " * p<.01$. 
Table 3

Self-Reports of Procedure Use Averaged Across Participants $(n=16)$ and for Procedure Users

\begin{tabular}{|c|c|c|c|c|c|c|c|}
\hline \multirow[b]{3}{*}{ Procedure } & \multirow{2}{*}{\multicolumn{3}{|c|}{ Overall }} & \multicolumn{4}{|c|}{ Procedure Users Only } \\
\hline & & & & \multirow[b]{2}{*}{$n$} & \multirow[b]{2}{*}{$\%$ Use $^{\mathrm{a}}$} & \multicolumn{2}{|c|}{ Range of $\%$ Use } \\
\hline & RT & $\%$ Use & $\%$ Error & & & Min & Max \\
\hline \multicolumn{8}{|c|}{ Multiplicationb } \\
\hline Retrieval & 955 & 59 & 4.6 & 15 & 62 & 30 & 100 \\
\hline Visualize & 1,286 & 15 & 5.7 & 7 & 36 & 2 & 86 \\
\hline Repeated addition & 1,086 & 7 & 0.0 & 9 & 12 & 2 & 24 \\
\hline Series & 1,155 & 6 & 1.7 & 8 & 13 & 2 & 36 \\
\hline Derived fact & 2,103 & 4 & 0.0 & 8 & 8 & 2 & 32 \\
\hline Other & 1,072 & 7 & 6.2 & 9 & 13 & 2 & 30 \\
\hline \multicolumn{8}{|c|}{ Division } \\
\hline Retrieval & 1,157 & 45 & 1.4 & 14 & 51 & 9 & 100 \\
\hline Recasting & 1,312 & 37 & 16.6 & 15 & 40 & 2 & 98 \\
\hline Visualize & 1,484 & 4 & 0.0 & 6 & 12 & 2 & 42 \\
\hline Repeated addition & 1,161 & 4 & 2.6 & 6 & 11 & 2 & 37 \\
\hline Series & 1,046 & 1 & 0.0 & 3 & 5 & 2 & 11 \\
\hline Derived fact & 1,833 & 2 & 0.0 & 7 & 5 & 2 & 16 \\
\hline Other & 1,061 & 5 & 1.7 & 8 & 13 & 2 & 33 \\
\hline
\end{tabular}

aPercentage of use across individuals who used the procedure at least once. $\quad$ b On $2 \%$ of trials, no procedure was recorded due to experimenter error.

The percentage reported use of each multiplication procedure is shown in Table 3, across all trials (overall) and as an average for those participants who used each procedure. As in previous research (LeFevre, Bisanz, et al., 1996), retrieval was the most frequently reported procedure. However, the percentage reported use of retrieval was lower than in LeFevre, Bisanz, et al. (1996), mainly because the visualization procedure was reported frequently. LeFevre, Bisanz, et al. provided specific examples of each procedure (retrieval, repeated addition, and derived facts) in the instructions given to their participants, whereas, in the present experiment, specific examples of procedures were not given. Under the latter instructional conditions, it appears that participants phenomenologically distinguish between "knowing" the answer and visualizing their multiplication knowledge to extract the answer. The other reported procedures, such as series and derived facts, showed a similar frequency as in LeFevre, Bisanz, et al. (1996). Latencies across procedures were also similar to those in previous research: Retrieval was the fastest procedure, and derived facts were slow.

To assess whether the pattern of self-reports across problems was similar to that found in previous work, several regression analyses were conducted. Median latencies were regressed on the percentage use of retrieval, product, 5-operand status, and tie status (see Table 4). LeFevre, Bisanz, et al. (1996) found that percentage use of retrieval and product both explained a significant percent of the variance in multiplication latencies averaged over procedures. As shown in Table 4, the same pattern was found in the present experiment. Furthermore, analyses of Campbell's (1997) latencies showed essentially the same pattern (see Table 4; data for these analyses were obtained from the appendices provided by Campbell, 1997). Thus, as in previous research, latencies were related to the extent to which retrieval was reported on each problem.

Division. As shown in Table 3, self-reports of division procedures could generally be classified in a similar

Table 4

Unstandardized Coefficients for Multiple Regressions

of Median Latencies (in Milliseconds) and Percentage Use of Retrieval Across Problems on Structural Variables

\begin{tabular}{|c|c|c|c|c|c|c|}
\hline \multirow[b]{2}{*}{$\begin{array}{l}\text { Dependent } \\
\text { Measure }\end{array}$} & \multirow[b]{2}{*}{ Intercept } & \multicolumn{4}{|c|}{ Predictor Variables } & \multirow[b]{2}{*}{$R^{2}$} \\
\hline & & $\begin{array}{c}\text { Product } \\
\text { (Dividend) }\end{array}$ & 5-operand & Tie & $\begin{array}{c}\text { Percentage } \\
\text { Retrieval }\end{array}$ & \\
\hline \multicolumn{7}{|c|}{ Present Data } \\
\hline $\begin{array}{l}\mathrm{RT}_{\text {mult }} \\
\mathrm{RT}_{\text {div }}\end{array}$ & $\begin{array}{r}861.7^{* *} \\
1,007.1^{* *}\end{array}$ & $\begin{array}{l}8.2^{* *} \\
6.2^{* *}\end{array}$ & $\begin{array}{l}15.5 \\
58.6\end{array}$ & $\begin{array}{l}146.1^{*} \\
169.0^{* *}\end{array}$ & $\begin{array}{l}-3.0^{\mathrm{a}} \\
-3.7^{*}\end{array}$ & $\begin{array}{l}.52^{* *} \\
.58^{* *}\end{array}$ \\
\hline \multicolumn{7}{|c|}{ Campbell (1997) Data } \\
\hline $\begin{array}{l}\mathrm{RT}_{\text {mult }} \\
\mathrm{RT}_{\text {div }}\end{array}$ & $\begin{array}{r}861.6^{* *} \\
1,052.7^{* *}\end{array}$ & $\begin{array}{l}3.0^{* *} \\
2.4^{* *}\end{array}$ & $\begin{array}{l}51.6^{*} \\
80.3^{*}\end{array}$ & $\begin{array}{l}121.4^{* *} \\
138.4^{* *}\end{array}$ & $\begin{array}{l}-1.9^{* *} \\
-3.3^{* *} \\
\end{array}$ & $\begin{array}{l}.60^{* *} \\
.46^{* *} \\
\end{array}$ \\
\hline
\end{tabular}


way as multiplication procedures, with one exception. On a substantial number of trials, the participants reported accessing multiplication facts to solve division problems. We termed this procedure recasting because it was often described as translating a division problem, such as $56 / 7=$ ?, to a related multiplication format, $7 \times ?=56$. Some participants reported recasting as visualizing the multiplication problem, and others reported simply that they "thought of" the associated multiplication fact. The participants also reported using repeated addition (e.g., $12 / 4$ as $4+4+4$ ), derived facts (e.g., for $48 / 8,5 \times 8=$ 40 , so $6 \times 8=48$ ), and a variety of "other" procedures, such as recognizing special problems (e.g., ties as squares) or visualizing groups of dots or sticks. In general, procedures other than direct retrieval that were reported for division problems involved access to some form of multiplicative knowledge (e.g., recasting, repeated addition, or a multiplication-derived fact).

The self-reports of recasting are consistent with the hypothesis that division solutions are sometimes mediated through multiplication knowledge. Further examination of the use of recasting across problems supported the view that division knowledge was most likely to be supplemented with multiplication on the most difficult problems. We calculated the percentage reported use of recasting and retrieval for each of the 64 problems. The correlation between the percentage reported use of recasting and problem size (indexed by the dividend) was $r(62)=.55, p<.01$. As shown in Table 4, both dividend and percentage use of retrieval accounted for significant unique variance in latencies for division problems.

Summary. Self-reports of procedure use on multiplication problems were similar to those in previous research, although the participants appeared to distinguish between memory retrieval and access involving a visualization of a mental table. Self-reports on division problems supported the hypothesis that the participants often used multiplication knowledge to solve division problems. As in previous research, self-reports of procedures other than direct retrieval were more likely on larger problems than on smaller problems.

\section{Classification of Errors}

The total number of errors made by the participants in the present study (135 and 184 for multiplication and division, respectively) was quite small, so our analyses were limited to evaluation of whether our data produced a similar pattern of results to those of Campbell (1997). Errors were categorized into three mutually exclusive groups, prioritized as follows. First, answers that would have been correct for another operation were classified as operation errors. For multiplication, these were mainly addition or division errors to ties (e.g., $3 \times 3=6 ; n=5$ ). For division, all the operation errors in the present research involved confusion with subtraction (e.g., $6 / 3=3$; $n=6$ ). Second, answers in which one or both of the operands occurred were classified as naming errors. For multiplication, naming errors included errors where the left operand appeared as the decade digit (e.g., $4 \times 8=$ 42) or where the right operand appeared as the unit digit (e.g., $4 \times 8=38 ; n=31$ ). For division, a naming error occurred when the divisor was stated as the answer (e.g., $56 / 8=8$ ) or when either digit from the dividend appeared as the answer (e.g., $56 / 8=6 ; n=59$ ). Third, all remaining errors were classified as "other" but were grouped by problem set--that is, whether the larger operand was on the left or the right for multiplication or whether the divisor was larger or smaller than the quotient for division.

Campbell (1997) focused on three findings in his description of error types. First, he reported more operation errors for division than for multiplication ( $9 \%$ vs. $5 \%$ ). In contrast, percentage of operation errors in the present research was similar across operations ( $3 \%$ vs. $4 \%$ ). Second, Campbell (1997) reported more naming errors for division than for multiplication ( $39 \%$ vs. $23 \%$ ). The participants in the present experiment also made more naming errors in division than in multiplication $(32 \%$ vs. $23 \%)$, but the difference was not statistically significant $\left[\chi^{2}(1\right.$, $N=319)=3.34]$. Third, Campbell (1997) reported more errors on division problems when the divisor was smaller than the quotient (i.e., min-divisor) than when the divisor was larger than the quotient (i.e., max-divisor), whereas multiplication errors were not related to whether the smaller operand occurred first or second. We found a similar pattern, such that the participants made considerably more min-divisor errors than max-divisor errors on division problems (65\% vs. $35 \%)\left[\chi^{2}(1, N=110)=\right.$ 9.31]. According to Campbell (1997), min-divisor errors (e.g., $36 / 4=8$ ) are more likely than max-divisor errors (e.g., $36 / 9=3$ ) because the product implied by the incorrect answer on min-divisor problems (e.g., 32) is closer to the dividend than the implied product on max-divisor problems (e.g., 27). He argues that this finding supports the view that "division and multiplication performance were mediated by parallel or common associative structures" (Campbell, 1997, p. 1149). Because the absolute number of errors was quite small in the present study, we hesitate to draw strong conclusions from our data.

\section{DISCUSSION}

In the present research, we hypothesized that multiplication and division are stored in independent representations (Campbell, 1997; Rickard \& Bourne, 1996; Rickard et al., 1994) but that solution of division problems is sometimes mediated by direct access to multiplication knowledge. Two findings supported this hypothesis. First, the participants reported using multiplication to solve division problems on a substantial proportion of trials. In particular, they claimed to recast division problems as multiplication (e.g., $56 / 7=$ ? as $7 \times ?=56$ ). Second, multiplication performance benefited from prior solution of division problems more than division benefited from prior solution of multiplication problems. Furthermore, recasting was more likely to be reported on large division problems than on small division problems, and priming 
was greater on large multiplication problems than on small multiplication problems. We interpret these findings as evidence that recasting a division problem results in the direct activation of the multiplication representation. In contrast, if multiplication and division were solved via a common representation, priming should be symmetrical, and no differences in priming should be found across problem size.

Campbell (1997) argues that solvers use multiplication to check or validate the answer produced on each division trial. In contrast, we propose that the answer produced on some large-number division trials is being directly accessed from a multiplication representation during the solution process. Both views are consistent with the independent representation hypothesis; however, the mediation view suggests that the division representation may actually be incomplete for some problems, whereas the checking view suggests that division problems are always solved through accessing the division representation. Campbell (1997) provides three reasons for rejecting the mediation hypothesis. First, he suggests that if the multiplication check occurred before participants responded, error rates across operations would be more similar than he observed (i.e., approximately twice as many errors in division as in multiplication). In our data, error rates were greater for division than for multiplication, but not as dramatically different as was observed by Campbell (1997) and not significantly different when 2 extremely errorprone participants were omitted from the analysis. The substantial difference in error rates across operations found by Campbell (1997) may be an artifact of the mixed block methodology. Specifically, the weaker operation may be more disadvantaged by operation confusions than the stronger operation. Further empirical work is necessary to determine whether effects of blocking operation can account for these differences.

Second, Campbell (1997) suggests that the problem size effect should diverge for multiplication and division if access to multiplication information occurred before the solution was produced. Although not stated explicitly by Campbell (1997), a divergence is most likely if the multiplication access occurred more often on larger division problems than on smaller division problems. Campbell (1997) does not directly test whether the problem size effect does diverge across operations in the participantbased analyses, although he does not find evidence for divergence in the problem-based analyses. In particular, the slope of the regression of division latency on multiplication latency was 1.1 , not significantly different from 1.0. In the present data, we did find evidence for a divergence of the problem size effect across operations in the participant-based analysis by family (see Figure 1). Furthermore, in the problem-based analysis, the slope of the simple regression of division latency on multiplication latency was 0.82 , which was significantly different from $1.0[t(34)=-2.26, p<.05]$. When dividend, tie, and 5-operand status were added as predictor variables in a multiple regression, dividend predicted additional vari- ance in division latencies beyond that accounted for by multiplication performance ( $2.4 \mathrm{msec}$ per dividend unit; $p<.05$ ). Thus, in present data, the problem size effect in division did diverge from that predicted by multiplication latencies. The pattern of results in the present experiment is consistent with the view that additional processing occurs mainly on difficult division problems.

Third, Campbell (1997) argues that there should be positive transfer from division to multiplication if the multiplication check occurred before the answer was produced. He did not test for transfer in his data, but he cited work by Rickard and colleagues (Rickard \& Bourne, 1996; Rickard et al., 1994) as evidence for the lack of transfer across operations. In contrast, we found clear evidence that solution of division problems facilitated multiplication performance (see Figure 1). We suggest that transfer does not occur in training studies because, after training on division problems, solution of such problems no longer involves access to multiplication. Consistent with the independent representation hypothesis, practice strengthens the mental representations only of the specific problems that were practiced (Rickard \& Bourne, 1996). Transfer will be observed in situations like those in the present study, however, on the assumption that the division representation is less accessible or incomplete for large problems relative to small problems. In conclusion, our results are consistent with the view that access to multiplication knowledge occurs as part of the solution process for some division problems. It is possible, however, that some experimental procedures might lead to checking (e.g., an emphasis on speed and long delays between trials), whereas other procedures might lead to mediation (e.g., an emphasis on accuracy rather than speed). Further research using different experimental procedures will be necessary to examine these possibilities.

In the present research, we did not address the issue of why there are such strong similarities between multiplication and division if they are stored in separate representations. Division is typically the last basic operation taught to children and is likely to be illustrated as the converse of multiplication (Thornton, 1978). Thus, it seems plausible that early alternative solutions to division problems would involve access to known multiplication facts. For example, to solve $24 \div 6$, a child might activate several multiplication problems in order to figure out the answer (e.g., $6 \times 2=12,6 \times 3=18,6 \times 4=24)$. More generally, multiplicative relations may form the underlying conceptual basis for division, independent of formal instruction. For example, Mulligan and Mitchelmore (1997) found that, before receiving formal instruction in division, children in Grades 2 and 3 used similar solution procedures on word problems involving multiplication and division, and these solutions involved multiplicative and additive relations.

In conclusion, the results of the present experiment support the hypothesis that the mental representation of division facts is independent of that for multiplication. Furthermore, as in previous research with addition and 
multiplication, solution of simple division problems appeared to involve a variety of mental procedures and did not consist solely of direct associative retrieval (LeFevre, Bisanz, et al., 1996; LeFevre, Sadesky, \& Bisanz, 1996).

\section{REFERENCES}

Arbuthnott, K., \& CAMPBell, J. I. D. (1996). Effects of operand order and problem repetition on error priming in cognitive arithmetic. Canadian Journal of Experimental Psychology, 50, 182-195.

ASHCRAFT, M. H. (1995). Cognitive psychology and simple arithmetic: A review and summary of new directions. Mathematical Cognition, 1, 3-34.

Campbell, J. I. D. (1991). Conditions of error priming in number-fact retrieval. Memory \& Cognition, 19, 197-209.

Campbell, J. I. D. (1994). Architectures for numerical cognition. Cognition, 53, 1-44.

Camprell, J. I. D. (1995). Mechanisms of simple addition and multiplication: A modified network-interference theory and simulation. Mathematical Cognition, 1, 121-164.

CAMPBELL, J. I. D. (1997). On the relation between skilled performance of simple division and multiplication. Journal of Experimental Psychology: Learning, Memory, \& Cognition, 23, 1140-1159.

Campbell, J. I. D., \& Clark, J. M. (1989). Time course of error priming in number-fact retrieval: Evidence for excitatory and inhibitory mechanisms. Journal of Experimental Psychology: Learning, Memory, \& Cognition, 15, 920-929.

Campbell, J. I. D., \& Graham, D. J. (1985). Mental multiplication skill: Structure, process, and acquisition. Canadian Journal of Psychology, 39, 338-366.

CAMpbell, J. I. D., \& TARLING, D. P. M. (1996). Retrieval processes in arithmetic production and verification. Memory \& Cognition, 24, 156-172.

French, J. W., Ekstrom, R. B., \& Price, I. A. (1963). Kit of reference tests for cognitive factors. Princeton, NJ: Educational Testing Service.

GEARY, D. C. (1996). The problem-size effect in mental addition: Developmental and cross-national trends. Mathematical Cognition, 2, 63-93.

Geary, D. C., Frensch, P. A., \& Wiley, J. G. (1993). Simple and complex mental subtraction: Strategy choice and speed-of-processing differences in younger and older adults. Psychology \& Aging, 6, 474-483.

Geary, D. C., Widaman, K. F., \& Littre, T. D. (1986). Cognitive addition and multiplication: Evidence for a single memory network. Memory \& Cognition, 14, 478-487.

Geary, D. C., \& WiLEY, J. G. (1991). Cognitive addition: Strategy choice and speed-of-processing differences in young and elderly adults. Psychology \& Aging, 6, 474-483.

LeFevre, J., Bisanz, J., Daley, K. E., Buffone, L., Greenham, S. L., \& SADESKY, G. S. (1996). Multiple routes to solution of single-digit multiplication problems. Journal of Experimental Psychology: General, 125, 284-306.

LeFevre, J., \& LiU, J. (1997). Numerical cognition: Single-digit multiplication skills of adults from China and Canada. Mathematical Cognition, 3, 31-62.

LeFevre, J., SAdesky, G. S., \& Bisanz, J. (1996). Selection of procedures in mental addition: Reassessing the problem size effect in adults. Journal of Experimental Psychology: Learning, Memory, \& Cognition, 22, 216-230.

LeFevre, J., Smith, B. L., Hiscock, K., Daley, K. E., \& Morris, J. (in press). Young adults' strategy choices in simple arithmetic: Implications for the development of mathematical representations. In A. J. Baroody \& A. Dowker (Eds.), The development of arithmetic concepts and skills: Recent research and theory. Hillsdale, NJ: Erlbaum.

McCloskey, M., \& MaCaruso, P. (1994). Representing and using numerical information. American Psychologist, 50, 351-363.

Miller, K. F., Perlmutter, M., \& Keating, D. (1984). Cognitive arithmetic: Comparison of operations. Journal of Experimental Psychology: Learning, Memory, \& Cognition, 10, 46-60.
Mulligan, J. T., \& Mitchelmore, M. C. (1997). Young children's intuitive models of multiplication and division. Journal for Research in Mathematics Education, 28, 309-330.

Rickard, T. C., \& Bourne, L. E., JR. (1996). Some tests of an identical elements model of basic arithmetic skills. Journal of Experimental Psychology: Learning, Memory, \& Cognition, 22, 1281-1295.

Rickard, T. C., Healy, A. F., \& Bourne, L. E., JR. (1994). On the representation of arithmetic facts: Operand order, symbol, and operation transfer effects. Journal of Experimental Psychology: Learning, Memory, \& Cognition, 20, 1139-1153.

THORNTON, C. A. (1978). Emphasizing thinking strategies in basic fact instruction. Journal for Research in Mathematics Education, 9, 214227.

\section{NOTES}

1. There are two types of error priming: negative and positive. Negative error priming occurs within one trial of the target error; the most recently produced answer is less likely to occur as an error than expected by chance. Positive error priming occurs in the range of 2-10 previous problems. The answers to these problems are more likely to occur as an error to the target problem than expected by chance. Campbell (1997) did not observe significant negative error priming.

2. Data from the questionnaire are not reported here. See LeFevre, Smith, Hiscock, Daley, and Morris (in press).

3. Two individuals, both in the no-report condition, made a substantially greater number of errors on division problems than anyone else (means of $27 \%$ and $30 \%$, respectively). When the percent error data were reanalyzed, omitting these 2 individuals, the only significant effect was for operand families $\left[F(7,182)=13.13, M S_{\mathrm{e}}=59.7\right]$. Thus, apparent effects of report condition (and, incidentally, of operation) on percent error appeared to be carried by the performance of 2 particularly error-prone individuals.

4. Simple correlations between ties and latencies were also lower in the present research than in Campbell (1997). As calculated from the data in the appendices of that paper, the correlations between tie status and latencies were $r(62)=-.37$ and -.27 for multiplication and division, respectively.

5. Considerable theorizing has been directed toward the latency and error advantage observed for 5-operand problems (Campbell, 1995; LeFevre, Bisanz, et al., 1996; LeFevre \& Liu, 1997). One striking difference between division and multiplication in the latency data reported by Campbell (1997) was that there appeared to be an advantage on 7 . operand problems for division that was of a similar magnitude to the 5 operand advantage (see Campbell, 1997, Figure 1, p. 1145). As shown in Figure 1 of the present paper, a similar pattern was evident: Problems with operands of 7 were solved more quickly than problems with operands of 6, although the effect was not as dramatic as that observed by Campbell. To assess whether the 7-operand advantage was significant, we analyzed the division latencies presented in Appendix B of Campbell (1997) using the same regression model described above (see Table 2). Next, we added an additional predictor, 7-operand status (coded -.5 for 7 -operand problems, .5 for problems without operands of 7) to the regression equation. In the analysis of division latencies, 7 operand status accounted for a significant unique amount of variance, with a coefficient of $91 \mathrm{msec}$ (vs. a coefficient of $117 \mathrm{msec}$ for 5 operand status and $172 \mathrm{msec}$ for ties). In multiplication, 7-operand status did not account for significant unique variance $(p=.32)$. Thus, in Campbell's (1997) data, there was a 7-operand advantage for latencies. We also conducted the same analysis on the division data from the present study. The coefficient for 7 -operand status was $76 \mathrm{msec}$, but the unique contribution was not statistically significant $(p=.09$ ). This intriguing dissimilarity across operations on 7 -operand problems invites further research.

(Manuscript received February 6, 1998; revision accepted for publication September 25, 1998.) 